\title{
Evolución de la robótica social y nuevas tendencias
}

\author{
Antonio J. Pérez Vidal Álvaro Castro-González Fernando Alonso-Martín \\ University Carlos III of Madrid University Carlos III of Madrid University Carlos III of Madrid \\ 100355616@alumnos.uc3m.es_acgonzal@ing.uc3m.es_famartin@ing.uc3m.es
}

\author{
José Carlos Castillo Miguel Ángel Salichs \\ University Carlos III of Madrid University Carlos III of Madrid \\ Resumen \\ jocastil@ing.uc3m.es \\ salichs@ing.uc3m.es \\ aunque la mayoría de las personas piensen que
}

La robótica cada día es una realidad más presente en nuestras vidas, cambiando la forma con la que las personas interactuamos con nuestro entorno e incluso entre nosotros. En este artículo se se hace una revisión histórica del campo de la robótica social así como sus aplicaciones y campos de investigación. Para ello se muestra la evolución en cuatro periodos comprendiendo el origen, el estado actual de las investigaciones y el futuro sofisticado hacia el cual se dirige.

Palabras clave: Robótica social, interacción humano-robot, inteligencia artificial.

\section{INTRODUCCIÓN}

La ciencia ficción ha jugado un papel muy importante en la creación de la imagen de un robot. Una imagen presente en gran medida tanto en la industria cinematográfica como en la literatura. Este conjunto ha creado una idea parcialmente mitificada de lo que un robot es o puede llegar a ser. Sin embargo, el aludir a la idea de robot acuñada por la ciencia ficción como eso mismo (ciencia ficción), no significa que sea una idea descabellada o que esté tan alejada de la realidad, $o$ al menos de una realidad futura.

El concepto de robot difiere entre algunos científicos a la hora de adjudicarlo en algunas máquinas, pero generalmente todos coinciden en determinados factores. Así, un robot es una máquina programable que puede manipular objetos y realizar operaciones que antes sólo podían realizar los seres humanos. El robot puede ser tanto un mecanismo electromecánico físico como un sistema virtual de software. Ambos coinciden en brindar la sensación de contar con capacidad de pensamiento o resolución, aunque gran parte de los robots por ahora se limitan a ejecutar órdenes dictadas por las personas [17].

Nos encontramos en una época en la que la tecnología avanza a velocidades vertiginosas, existe cierto estancamiento en este ámbito. La realidad es que el mundo de la ciencia y la tecnología sí que ha avanzado a grandes pasos en estas décadas. Hace cuarenta años nadie podía soñar con poder hablar con alguien a distancia y al mismo tiempo poder ver a esa persona, y sin embargo ahora tenemos smartphones que hacen eso y mucho más. Tenemos entre otras muchas cosas, mecanismos de reconocimiento facial y de voz, comienzan a aparecer los coches autónomos, e incluso se está organizando actualmente el viaje de colonización a Marte. Cosas que hoy en día parecen prácticamente normales y sin embargo años décadas atrás tan sólo eran sueños.

Dentro de toda esta nueva era tecnológica la robótica juega un papel fundamental, y es que su estudio aporta cada vez más mejoras a la vida cotidiana y a los trabajos complicados. Campos como los exoesqueletos estudian ayudar a las personas con discapacidades motrices, intentando mejorar sus vidas; los automóviles autónomos se encuentran ya en el mercado comenzando a aumentar sus ventas; los robots espaciales nos brindan imágenes de planetas a miles de millones de kilómetros de distancia y nos ayudan a poder entender mejor nuestro universo.

Aquí tienen un lugar especial los denominados robots sociales cuyo objetivo principal es interactuar con los humanos de forma totalmente natural e intuitiva como si fueran un ser vivo más. Vamos a entrar en materia dentro de la robótica social, haciendo un repaso acerca de en qué consiste exactamente un robot social y comentando brevemente los orígenes de esta ciencia, su estado actual y por último un breve apartado imaginativo sobre qué deparará el futuro de esta tecnología.

\section{ROBÓTICA SOCIAL}

La robótica social está introduciéndose a un ritmo moderado dentro de la sociedad humana formando parte de la normalidad del día a día, permitiendo así que el contacto y la interacción 
humano-robótica se produzca a mayor escala, incluyendo así poco a poco a los robots en la vida social humana. Pero, ¿qué quiere decir que un robot es social? La sociabilidad implica la existencia de relaciones de interacción. Un agente capaz de interactuar y poseer un comportamiento comunicativo es considerado social. La simple existencia de dos robots autónomos en el mismo entorno fuerza aspectos del contacto social, sea éste directo o indirecto.

Una perspectiva es que uno mismo debe estar consciente de sus estados mentales y ser capaz de atribuir estados mentales a otros, lo que permite predecir y analizar los comportamientos de uno mismo y de los demás. Esto permitiría a uno ser capaz de lidiar con relaciones sociales complejas y también exhibir la habilidad para resolver problemas abstractos. La hipótesis Maquiavélica [2], promulga que todas estas capacidades intelectuales evolucionaron de un dominio social. La hipótesis de inteligencia social fomenta la teoría de que para poder alcanzar un grado de comportamiento inteligente de un agente, éste debe estar incorporado tanto en un entorno físico como en un entorno social. Este agente estará por lo tanto sujeto a interacciones sociales dinámicas y complejas en el mundo real, factor que en Duffy, Brian R. et al. [2] es considerado necesario para el desarrollo de un agente de inteligencia artificial.

El entendimiento mutuo por parte de los miembros de un grupo social es necesario para el establecimiento y continuidad del grupo. Los problemas aparecen cuando miembros individuales están motivados por intereses exclusivamente propios. Sin embargo, en muchos grupos sociales aparecen grados de flexibilidad con la evolución de cada estatus social de los miembros, y puede ser diferente en distintos contextos orientados a objetivos. Mientras que en sociedades primitivas se utiliza solamente el contacto físico como interacción social, los humanos hemos desarrollado métodos más eficientes a través del lenguaje.

Se cree que el lenguaje no funciona tan sólo como un método para adquirir conocimiento sobre las características del comportamiento de otros, sino que también expone sus estados internos como los sentimientos y aptitudes. Para poder construir una base para la interacción y la cooperación, los individuos tienen que comunicarse y unir sus conceptos del mundo con los grados de abstracción siendo socialmente fundamentados y actualizados continuamente.

Como se puede comprobar, la inclusión de los robots sociales en términos de definición es un tema bastante delicado, puesto que es necesaria la existencia de una conjunción de una gran variedad de factores inherentes a la inteligencia para poder establecer un agente social. Los robots sociales aún se encuentran en un estadio precoz en cuanto a las consideraciones de la comunicación y la interacción, dependientes de la inteligencia artificial. Además de esos factores, deben poder ser autónomos y también poseer una apariencia agradable ya que su objetivo principal será la interacción con los humanos, también tienen que ser fáciles de programar por personas no expertas, intrínsecamente seguros, tolerantes a percepciones y acciones imprecisas y, sobre todo, estar dotados de una gran capacidad de aprendizaje y adaptabilidad a entornos no predefinidos y dinámicos. En términos básicos, se puede definir a un robot social como "aquel robot que interactúa y se comunica con las personas (de forma sencilla y agradable) siguiendo comportamientos, patrones y normas sociales"[19].

Aunque los robots aún se encuentran lejos de poder tomar consciencia propia y formar jerarquías basadas en comunidades sociales, su inclusión e interacción con la sociedad es cada vez más patente, promoviendo así su desarrollo y su investigación gracias a la aceptación social.

\subsection{Primer período (1940-1990)}

Podría decirse que en el inicio, la robótica social se inspiró en las investigaciones de las comunidades biológicas animales [8] y las interacciones entre los individuos que las componen. Los investigadores siempre se han fascinado por la naturaleza que les rodea y sus composiciones, intentando recrearlas de forma artificial en los laboratorios. Así nace la robótica social (en un estado primigenio) al intentar reproducir las interacciones de las comunidades biológicas de forma artificial.

Uno de los primeros experimentos acerca de la interacción de los robots con el entorno fue llevado a cabo por William Grey Walter [22], el cuál diseñó y construyó unas tortugas robóticas (Figura 1) en a finales de los cuarenta. Mediante faros enganchados en la parte frontal de la carcasa y fototaxis positiva, estas tortugas interactuaban de una forma similar a la social, aunque no había comunicación explícita o reconocimiento mutuo.

Mientras el campo de la vida artificial emergía, los investigadores empezaron a aplicar principios como la estigmergia [7] (comunicación indirecta entre individuos a través de modificaciones en el entorno compartido; en sistemas descentralizados, tales como las colonias de hormigas, los diferentes componentes colaboran a través de pautas o hitos dejados en el medio: feromonas, acumulación de 


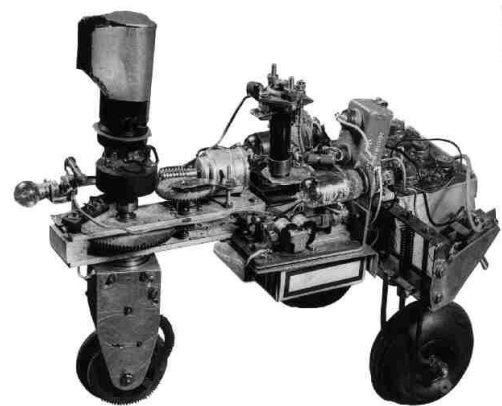

Figura 1: Tortuga robot Elmer de Walter [6]

objetos o cualquier otro tipo de cambio físico, como la temperatura) para lograr comportamientos colectivos en robots.

Uno de los pioneros en los primeros experimentos de la estigmergia fue Jean Louis Deneubourg, mediante la creación de robots parecidos a las hormigas en 1990 [8]. Desde entonces, una gran cantidad de investigadores han desarrollado robots colectivos y han usado robots como modelos para el estudio del comportamiento social de los insectos.

Principios parecidos se pueden encontrar en investigaciones sobre sistemas multi-robot o sistemas robóticos distribuidos. Algunos mecanismos de interacción utilizados son comunicación, interferencia y competición agresiva.

\subsection{Segundo Período (1990-2005)}

En 1990 se crea Kismet [12], uno de los primeros robots sociales. Kismet (Figura 2) es una cabeza robótica fabricada en el MIT por la doctora Cynthia Breazeal como un experimento en computación afectiva, una máquina capaz de reconocer y simular las emociones. Para interactuar adecuadamente con los humanos, posee dispositivos de entrada que le proveen de audición, visión y habilidades propioceptivas. Además, Kismet simula emociones mediante varias expresiones faciales, vocalizaciones y movimiento. La expresiones se crean gracias a los movimientos de las orejas, cejas, párpados, labios, mandíbula y cabeza.

La idea de la doctora Breazeal era representar su relación con el robot como lo hace un padre o un cuidador con su hijo.

Entre 1996 y 2001 la compañía japones Honda crea los robots antropomórficos P1, P2, P3, P4 y finalmente ASIMO (Figura 3). Aunque los cuatro primeros robots fueron creados para investigar sobre la robótica humanoide, ASIMO sirvió para investigar además con la interacción humano-robot, específicamente con los robots de compañía [10]. Para ello es capaz de reconocer las posturas y los gestos, pudiendo reaccionar a ellos, además distingue los sonidos, es decir, puede responder a su

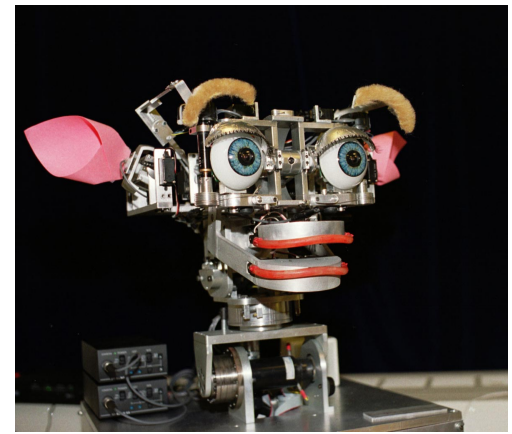

Figura 2: Robot Kismet [4]
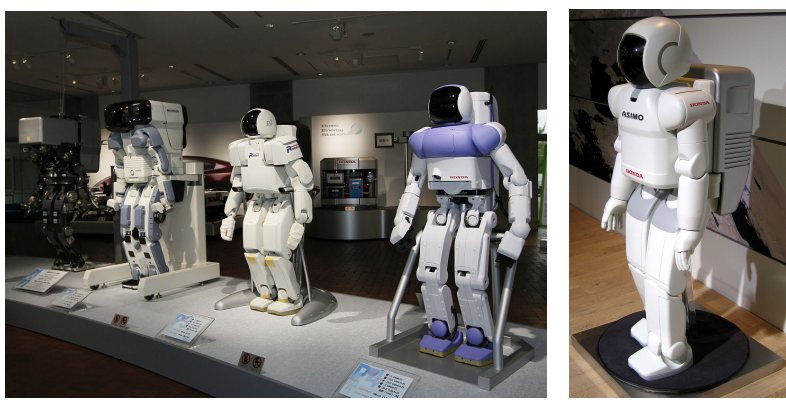

Figura 3: De izquierda a derecha: robots P1, P2, P3, P4 y ASIMO [11]

nombre, observar a la gente cuando se le habla, y reconoce sonidos inusuales como el de un objeto que cae o una colisión, y mira en la dirección del golpe. Es capaz de responder preguntas, mediante un breve movimiento del cuerpo en general o de solo la cabeza, o con una respuesta verbal.

A partir del siglo XXI, gracias a la nuevas tecnologías, la robótica social comienza a tener un sentido físico y tecnológico y empiezan a aparecer cada vez más robots sociales con capacidad de interacción con los humanos.

\subsection{Tercer período (2009-2017): Presente}

Se ha avanzado mucho desde aquellos robots sociales primigenios anteriormente comentados. La tecnología que actualmente está en manos de los investigadores supera con creces aquella de la que disponían los pioneros de la robótica. Con cada año que pasa se obtienen nuevos avances tecnológicos que proveen de mejores herramientas para enfocar todo tipo de investigaciones.

A lo largo de estos años, se han llevado a cabo numerosas investigaciones en una gran variedad de ámbitos dentro de la robótica social. Una parte importante de la interacción humano-robot es la representación de emociones. Diversos estudios han demostrado que un robot puede imitar 


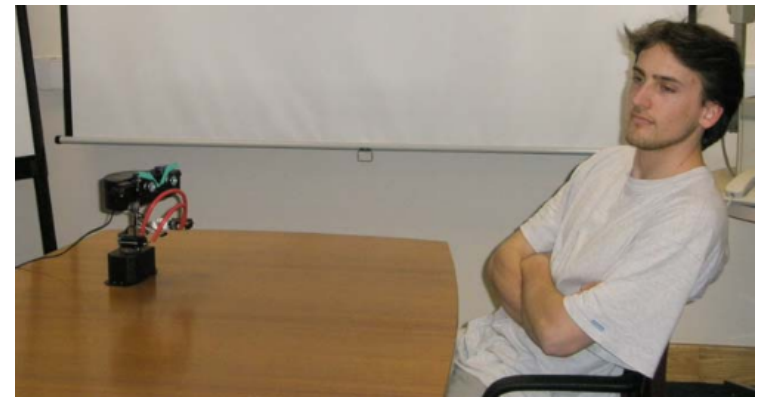

Figura 4: El robot ERWIN falla al intentar atraer la atención del usuario, por lo que expresa enfado después de varios intentos fallidos [16].

expresiones humanas reconocibles. Es necesario tanto que el humano sea capaz de reconocer la expresión del robot analizando el contexto en el que se entra, como viceversa, ya que las respuestas emocionales de ambos agentes se deben adecuar a lo que sus sentidos perciben. Para avanzar en este propósito se han realizado experimentos con robots que tenían un rango de expresiones (alegría, tristeza, enfado, sorpresa y miedo) que mostraban a una persona para atraer su atención [16]. El robot reconocía la cara del humano y dependiendo de si la persona le prestaba atención o no, iba variando sus expresiones (4). Por supuesto además de la expresión reconocida, el contexto emocional en el cual se produce es muy importante para entenderla, ya que aunque las expresiones de los robots están bastante logradas, en algunas ocasiones pueden confundir al usuario.

En el experimento realizado en Zhang J. y Sharkey A.J.C. [24], se investigó la capacidad de interpretación de los usuarios ante las expresiones de un robot con un contexto musical, tanto cuando éstas eran congruentes e incongruentes con él. Se comprobó así que un contexto musical afecta a la forma de juzgar las expresiones del robot.

Además de reconocer los gestos y expresiones de los robots, el usuario debe sentirse cómodo con el robot. El estudio del aspecto de los robots es esencial para que las interacciones sociales se puedan llevar a cabo. Los humanos somos una especie con costumbres arraigadas y con temor a lo desconocido. Todo aquello que nos resulta extraño y poco o nada familiar nos produce una sensación de desconfianza y miedo, por lo tanto si un robot tiene unos rasgos poco familiares o un tanto oscuros y extravagantes, probablemente produzca una reacción de rechazo en la mayoría de las personas. Es por ello por lo que los robots sociales tienen formas familiares, para que las personas se

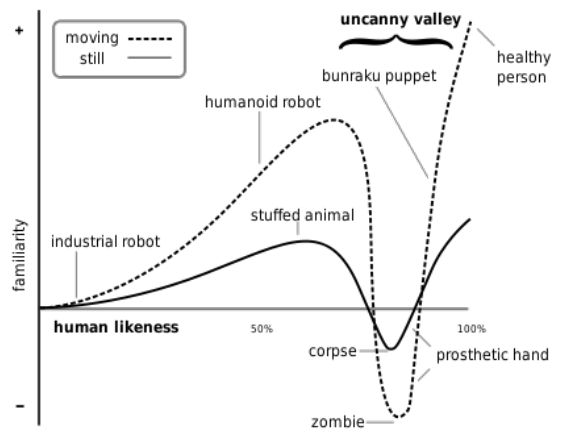

Figura 5: Diagrama del Uncanny Valley [20]

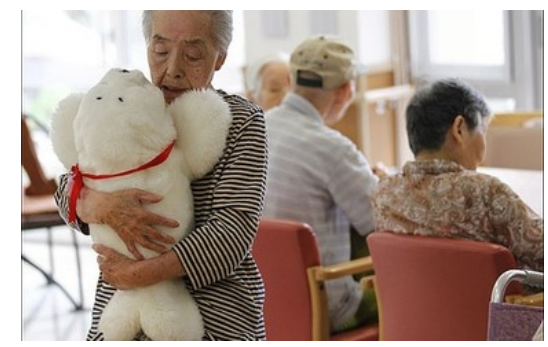

Figura 6: Robot Nuka como entretenimiento beneficioso para ancianos [18]

sientan cómodas con ellos y no se asusten. Para ello se les dota a los robots de ciertos rasgos humanos como ojos, boca, cejas, orejas. Sin embargo estudios realizados sobre la apariencia humana de los robots demuestran que cuanto más cerca de la apariencia real humana se encuentra el robot, más rechazo produce. Este fenómeno es el denominado uncanny valley, cuya curva de agradohumano se puede apreciar en la Figura 5. Aparte de la apariencia se ha observado que el audio también influye en generación de la sensación de extrañeza y repulsión. En experimentos realizados en Meah L.F.S. y Moore R.K. [15], la apariencia realmente humana de robots junto con voces humanas mezcladas electrónicamente provocaban el rechazo. Por un lado los ojos eran un factor determinante en la apariencia ya que se consideraban como ojos sin luz y sin vida. Por otro lado la voz humana modificada electrónicamente producía una tensión perceptiva que era considerada como extraña y horrible.

Otros estudios se centran en el uso de los robots sociales en el campo de la asistencia a ancianos y niños. Los experimentos llevados a cabo en este campo comprenden desde robots que ayudan a personas con enfermedades degenerativas, como el Parkinson, a tomar su medicación [23], robots con formas de animales para la mejora de la salud de los ancianos [13] (Figura 6), etc.

Un ejemplo de un experimento de este tipo es el llevado acabo en Fujimoto I. et al. [9], donde un 


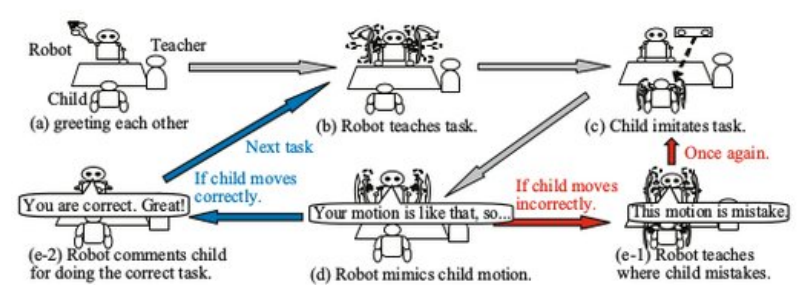

Figura 7: Escenario interactivo de imitación [9]

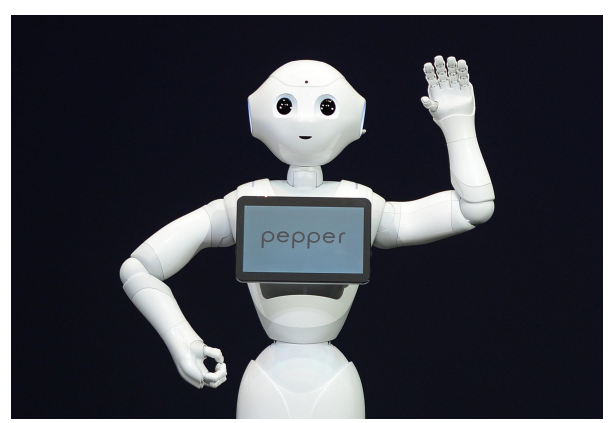

Figura 8: Robot Pepper [5]

robot social producía movimientos que el niño con autismo debía copiar, el robot observaba al niño y si éste se equivocaba le corregía hasta que el resultado fuera el buscado. Los resultados fueron satisfactorios, demostrando que los niños realmente mejoraban sus habilidades de imitación. El método de interacción se puede observar en la Figura 7 .

Además de todos los estudios y experimentos que se están realizando, se pueden destacar varios robots sociales conocidos que están a la vanguardia de la tecnología.

El primero y el cual ya se está comercializando, se trata del robot Pepper (Figura 8). Creado por SoftBank Robotics [21]. Su principal cualidad es la de percibir las emociones y adaptar su comportamiento al estado anímico del usuario. En Japón este robot se está utilizando ya como forma de recibir, informar y entretener a los clientes.

Dentro del ámbito de robots sociales de entretenimiento educativo y asistencial podemos encontrar al robot MAGGIE (Figura 9). MAGGIE [14] ha sido diseñado y creado en el departamento de robótica social del Robotics Lab de la Universidad Carlos III de Madrid. Su objetivo principal es el de estudiar la interacción humano-robótica, además de avanzar en el desarrollo de la autonomía y la inteligencia de los robots.

Además de MAGGIE, el Robotics Lab también posee los robots Mini Maggie y MBot (Figura 10), el robot con el que participaron en el proyecto europeo $\mathrm{MOnarCH}$, que se utilizan en proyectos de robótica social con ancianos, personas dependien-

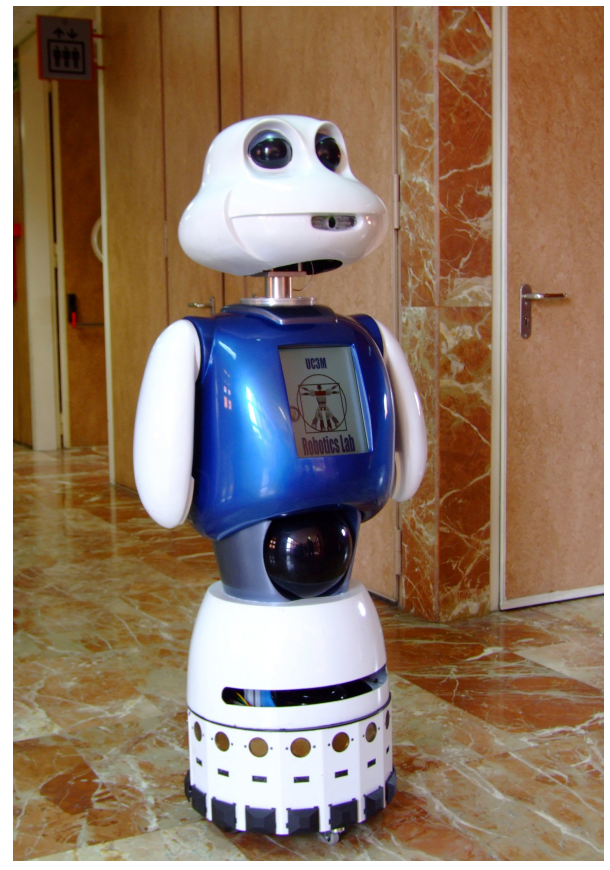

Figura 9: Robot MAGGIE [14]

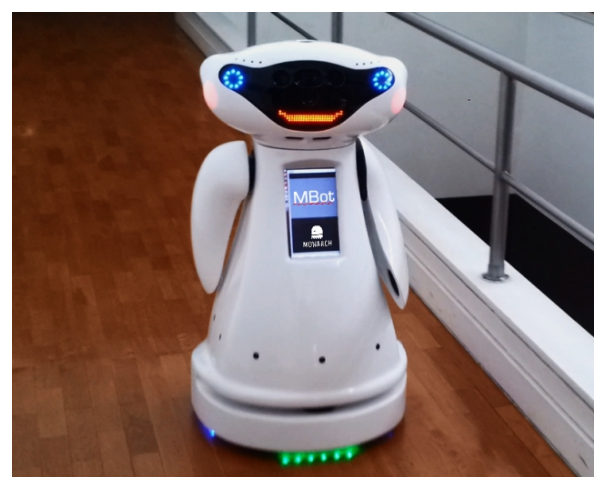

Figura 10: Robot MBot [3]

tes y niños ingresados en hospitales.

La lista de robots sociales es cada vez más larga puesto que cada vez más este tipo de robots se ve como proyectos comerciales. Entre ellos podemos encontrar a NAO, el robot HRP-4C, Nuka, Jibo o Sota que también abarcan el campo de asistenciales.

\subsection{Futuro}

En un futuro próximo los avances dentro de la robótica social nos mostrarán varios tipos de robots totalmente desarrollados tecnológicamente como por ejemplo los robots asistenciales que ayudarán en los hospitales, o en las casas de las personas discapacitadas o enfermas. Facilitarán las labores sanitarias y ayudarán acelerar las actividades de urgencias e incluso en planta. Además podrán ayudar a los médicos a evaluar un 
diagnóstico cuando sea requerido ya que tendrán una base de datos a la que podrán acceder con suma rapidez, con todas las enfermedades y sus respectivas curas. Por supuesto existirán versiones robóticas de ayuda en cirugía, más avanzadas que las que están empezando a surgir. Las incluyo dentro del ámbito de los robots sociales porque se relacionarán con el cirujano aconsejándole y compartiendo y aliviando así la carga de la operación.

En cuanto a los robots que asistan en casas de personas discapacitadas o enfermas, dispondrán de la tecnología necesaria para administrar los fármacos y terapias prescritas por sus médicos, además de ayudarles con sus tareas diarias.

Por otro lado, tanto en las residencias de ancianos como en las plantas pediátricas los pacientes podrán disponer y disfrutar de la compañía de robots sociales que les entretengan y les diviertan jugando y hablando con ellos, haciendo su día a día un poco más llevadero. Todos estos robots no tendrán por qué ser necesariamente antropomorfos, si no que habrá modelos con diferentes formas que corresponderán a las necesidades que se requieran de ellos.

Todos los robots sociales deberán estar provistos de una inteligencia artificial, que les permita aprender de su entorno y poder reaccionar a diferentes situaciones del modo correcto. Esta inteligencia será la que les dote de su autonomía. Naturalmente con los años esta inteligencia será más sofisticada, hasta el punto que los robots realmente puedan tomar sus propias decisiones sin necesidad de que un humano deba decirles qué hacer en cada situación.

Sin embargo, si con inteligencias artificales mejoradas adquieren una mayor autonomía, si aprenden más acerca de todo lo que les rodea, incluso de sí mismos, llegado el momento, ¿es posible que pudieran llegar a adquirir consciencia propia? ¿Es posible que lleguen a razonar? Y si esto ocurriera, ¿no podrían ser considerados entonces como seres con "vida" de algún modo? Si un robot es capaz de razonar y de pensar, entonces puede que llegue a comprender su existencia y qué lugar ocupa en la sociedad, pudiendo por lo tanto definirse como un individuo. Quizá llegado este punto, en el que toma consciencia de que es una herramienta para la sociedad, pueda comenzar a desarrollar una especie de sentimientos, como ira o miedo hacia los humanos, quizá al poder pensar por sí mismo, afloren en él ciertas ansias de libertad, de poder decidir que hacer con esta especia de vida con la que se la ha dotado. Es aquí donde entrarán en conflicto multitud de valores éticos como el esclavismo del que hablábamos antes, apelando a dere- chos como la vida o la seguridad.

El artículo 3 de la Declaración de los Derechos Humanos define: Todo individuo tiene derecho a la vida, a la libertad y a la seguridad de su persona. Si el robot en cuestión puede definirse a sí mismo como un individuo diferente a otros robots, siendo único, ¿no puede ser aplicado este artículo a su favor?

Evidentemente estos son terrenos pantanosos de difícil salida, y tendríamos que obviar el hecho de que este artículo se aplica dentro de los derechos humanos y un robot definitivamente no es un ser humano.

No obstante, si algo nos ha enseñado la historia es que ante los nuevos cambios, siempre existen dos bandos, uno a favor y otro en contra, por lo que en este caso habría gente prorrobótica y gente robofóbica. Habría personas que lucharían por la declaración de unos derechos robóticos, rogando por el reconocimiento de su ser y su libertad.

Además, como explicábamos antes en lo referente al tema del parecido físico a los humanos, en este caso la cosa cambia. Si antes la semejanza producía un rechazo, ahora se producen dos vertientes.

Al demostrar su propia consciencia y su capacidad de pensar y razonar, el segmento de la sociedad que sea prorrobótico, los aceptará con mayor fervor que antes, llegando incluso a producirse relaciones entre robots y humanos, pudiendo proclamarse estos últimos como robosexuales. Por otro lado la parte de la sociedad que sea robófoba, denotará un rechazo mayor, pronunciado por el temor a estos seres mecánicos que se hacen pasar por personas.

\section{CONCLUSIONES}

A lo largo del artículo se han presentado las nociones principales de la definición de robot, así como de la robótica social, mostrando en qué consiste exactamente esta materia y qué campos de acción abarca. Para poder englobar esta especialidad, se ha realizado un repaso a las etapas evolutivas de la robótica social, pudiendo así observar los progresos realizados en distintos campos y su difusión a tantos otros.

Como se ha podido comprobar la robótica social se encuentra cada vez más presente en nuestro uso diario, proporcionándonos día tras día servicios de atención al usuario. La interacción humano-robot está patente en multitud de campos, desde juguetes y atención al cliente hasta el tratamiento de pacientes, facilitando en gran medida las tareas de los trabajadores y aportando soluciones y beneficios a investigaciones tecnológicas y humanas, además de proporcionar un entretenimiento instructivo y divertido. 
El desarrollo y progreso de las distintas aplicaciones de la robótica social avanza a pasos agigantados hacia modelos más sofisticados procurando un futuro cercano lleno de tecnologías robóticas. Sin embargo, aunque por supuesto el progreso siempre es beneficioso, se tienen que tener en cuenta muchos factores a la hora de implantar una nueva tecnología y es por ello que al igual que la robótica social se encuentra en plena investigación, así mismo se encuentran las leyes que por las que se regirán las acciones procuradas por un entorno robótico sofisticado. Entran así en conflicto temas éticos como la influencia de un robot en la educación, en el crecimiento infantil, la moralidad esclavista en cuanto a máquinas, derechos robóticos, los factores psicológicos y emocionales que esta generación tecnológica puede producir, etc.

\section{Agradecimientos}

La investigación desarrollada ha recibido financiación de dos proyectos: "Development of social robots to help seniors with cognitive impairment" (ROBSEN), financiado por el Ministerio de Economía y Competitividad; y "RoboCity2030-IIICM", financiado por la Comunidad de Madrid y cofinanciado por los Fondos Estructurales de la Unión Europea.

\section{Referencias}

[1] Canal Jubilación (s.f.), Asibot y Maggie, dos robots para personas mayores "made in Spain", Jubilación $C A$ NALES MAPFRE. Recuperado de: https://www.jubilacionypension.com/planesfondos/asibot-y-maggie-dos-robots-parapersonas-mayores-made-in-spain/

[2] Duffy, Brian R., Rooney, Colm, O'Hare, Greg M.P., O'Donoghue, Ruadhan, (1999) What is a social robot?, 10th Irish Conference on Artificial Intelligence and Cognitive Science, University College Cork, Ireland, 1-3 September, 1999

[3] Edutainment, (s.f.), Idmind Living Robotics. Recuperado de: http://www.idmind.pt / markets/edutainment/

[4] Edwards, T., (2015), Emotive Robots Key to Unlocking IOT Potential, Digitally Aproved Blog. Recuperado de: http: //www.digitallyapproved.com/tag/kismet/

[5] El robot Pepper se agota en un minuto al salir a la venta en Japón, (2015), Público. Recuperado de: http://www.publico.es/ ciencias/robot-pepper-agota-minuto-al.html
[6] Elmer_Elsie Robots (s.f.), The Old Robots Website. Recuperado de: http://www .theoldrobots.com/ElmerElsie.html

[7] Estigmergia (s.f.), Wikipedia. Recuperado de: https://es.wikipedia.org/wiki/Estigmergia

[8] Fong, T., Nourbakhsh, I., Dautenhahn, K. (2003) A survey of socially interactive robots. In: Robotics and Autonomous Systems, Volume 42, Issues 3-4, Elsevier, Pittsburgh, Lausanne, Hertfordshire

[9] Fujimoto I., Matsumoto T., De Silva P.R.S., Kobayashi M., Higashi M. (2010) Study on an Assistive Robot for Improving Imitation Skill of Children with Autism. In: Ge S.S., Li H., Cabibihan JJ., Tan Y.K. (eds) Social Robotics. ICSR 2010. Lecture Notes in Computer Science, vol 6414. Springer, Berlin, Heidelberg

[10] George A. Bekey, (2005) Autonomous Robots: From Biological Inspiration to Implementation and Control, MIT Press, Hardcover, 577 pages. ISBN 0262025787

[11] Honda P Series (Robots) (s.f.), Wikipedia. Recuperado de: https://es.wikipedia.org /wiki/Honda_P_series_(Robots)

[12] Kismet (robot) (s.f.), Wikipedia. Recuperado de: https://en.wikipedia.org/wiki/Kismet -(robot)

[13] Leong T.W., Johnston B. (2016) Co-design and Robots: A Case Study of a Robot Dog for Aging People. In: Agah A., Cabibihan JJ., Howard A., Salichs M., He H. (eds) Social Robotics. ICSR 2016. Lecture Notes in Computer Science, vol 9979. Springer, Cham

[14] Maggie: futuro, autonomía y diversión (s.f.), uc3m, Universidad Carlos III de Madrid. Recuperado de: http://portal.uc3m.es/portal/actualidad_cientifica/actualidad/reportajes/archivo_reportajes/archivo_reportajes/Maggie_futuro_autonomia_diversion

[15] Meah L.F.S., Moore R.K. (2014) The Uncanny Valley: A Focus on Misaligned Cues. In: Beetz M., Johnston B., Williams MA. (eds) Social Robotics. ICSR 2014. Lecture Notes in Computer Science, vol 8755. Springer, Cham

[16] Murray J.C., Cañamero L., Bard K.A., Ross M.D., Thorsteinsson K. (2009) The Influence of Social Interaction on the Perception of Emotional Expression: A Case Study with a 
Robot Head. In: Kim JH. et al. (eds) Advances in Robotics. FIRA 2009. Lecture Notes in Computer Science, vol 5744. Springer, Berlin, Heidelberg

[17] Pérez Porto, J., Merino, M.. Publicado: 2010. Actualizado: 2013. Definición de robot, Definicion.de. Recuperado de: http://definicion.de/robot/

[18] Quincoces, A., (2016), La foca Nuka, un peluche robotizado que mejora la salud sin efectos secundarios, ELPROGRESO. Recuperado de: http://elprogreso.galiciae.com/ noticia/497120/la-foca-nuka-un-peluche-ro botizado-que-mejora-la-salud-sin-efectos-sec undarios

[19] Rafael Rivas E., Eladio Dapena G. (s.f.), Construcción de un Robot con Habilidades Sociales, LASDAI. Recuperado de: http://www.ing.ula.ve/lasdai/?page_id $=26$

[20] Uncanny Valley (s.f.), Wikipedia. Recuperado de: https://simple.wikipedia.org/wiki/ Uncanny_valley

[21] Who is Pepper? (s.f.), SoftBank Robotics. Recuperado de: https://www.ald.soft bankrobotics.com/en/cool-robots/pepper

[22] William Grey Walter (s.f.), Wikipedia. Recuperado de: https://es.wikipedia.org/wiki/ William_Grey_Walter

[23] Wilson J.R., Tickle-Degnen L., Scheutz M. (2016) Designing a Social Robot to Assist in Medication Sorting. In: Agah A., Cabibihan JJ., Howard A., Salichs M., He H. (eds) Social Robotics. ICSR 2016. Lecture Notes in Computer Science, vol 9979. Springer, Cham

[24] Zhang J., Sharkey A.J.C. (2011) Listening to Sad Music While Seeing a Happy Robot Face. In: Mutlu B., Bartneck C., Ham J., Evers V., Kanda T. (eds) Social Robotics. ICSR 2011. Lecture Notes in Computer Science, vol 7072. Springer, Berlin, Heidelberg 